\title{
UTILIZING MOMENT INVARIANTS AND GROBNER BASES TO REASON ABOUT SHAPES *
}

\author{
Haim Schweitzer \\ The University of Texas at Dallas \\ P.O. Box 830688 \\ Richardson, Texas 75083-0688 \\ haim@utdallas.edu
}

\author{
Janell Straach \\ The University of Texas at Dallas \\ P.O. Box 830688 \\ Richardson, Texas 75083-0688 \\ straach@utdallas.edu
}

\begin{abstract}
Shapes such as triangles or rectangles can be defined in terms of geometric properties invariant under a group of transformations. Complex shapes can be described by logic formulae with simpler shapes as the atoms. A standard technique for computing invariant properties of simple shapes is the method of moment invariants, known since the early sixties. We generalize this technique to shapes described by arbitrary monotone formulae (formulae in propositional logic without negation). Our technique produces a reduced Grobner basis for approximate shape descriptions. We show how to use this representation to solve decision problems related to shapes. Examples include determining if a figure has a particular shape, if one description of a shape is more general than another, and whether a specific geometric property is really necessary for characterizing a shape. Unlike geometry theorem proving, our approach does not require the shapes to be explicitly defined. Instead, logic formulae combined with measurements performed on actual shape instances are used to compute well characterized least squares approximations to the shapes. Our results provide a proof that decision problems stated in terms of these approximations can be solved in a finite number of steps.
\end{abstract}

\section{Introduction}

Like many natural language terms, the intuitive notion of a shape is not easily captured by a formal definition that can be translated into a computer program. For example, in grammar school we learn that a triangle is a polygon with three sides, and this definition can be easily applied to recognize two triangles in Fig. 1. Yet, a computer program that implements this definition needs to determine first what is a polygon, and what is a polygon side. This appears to be much harder.

\footnotetext{
* Research partially supported by NSF grant IRI-9309135
}
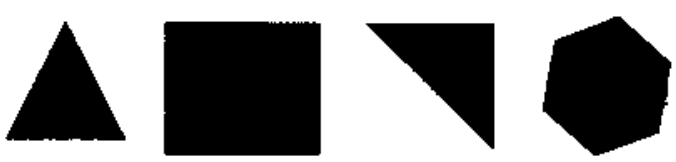

Figure 1: triangles and non-triangles

The commonly accepted formal (mathematical) definition of a shape relates the shape to properties invariant under a group of transformations. See Section 2 . This formal definition is independent of a language that may be used to describe the shape. Section 2.1 describes the method of moment invariants [Hu, 1962; Reiss, 1991] that gives a technique for transforming simple shape descriptions into a computer code. Unfortunately, this technique can only be applied to shapes that are completely characterized by a single instance, e.g., a triangle. In spite of its limitations the method of moment invariants was successfully used in many pattern recognition and image processing situations. (See, e.g., [Hu, 1962; Li, 1992; Wong and Hall, 1978]).

In this paper we generalize the method of moment invariants and describe a technique for computing invariants of shapes given by logic formulae. We view these invariants as shape descriptors and show how to use them in reasoning tasks. Allowing shapes to be described in a language (logic in our case) enables handling shapes that cannot be characterized by a single instance. (An example is a shape described as being either a triangle or a rectangle.) Our technique can be applied to arbitrary monotone formulae (formulae in propositional logic without negation).

When the method of moment invariants is applied in practice, approximate characterizations of simple shapes are computed from a small number of moment invariants. A straightforward approach of combining these invariants according to the relations expressed by a short monotone formula may produce a new set of invariants of size exponential in the original formula length. Many of these invariants may be redundant. As described in Section 4, the redundancy can often be eliminated by representing the set of invariants as a reduced Grobner base [Becker and Weispfenning, 1993; Buchberger, 1985]. This representation allows decision 
problems related to shapes to be easily solved. (The Grobner base algorithm guarantees that these problems are solved in a finite number of steps.) Unfortunately, it is known that there can be cases where a reduced Grobner base may contain a huge number of such invariants [Huynh, 1986], but experimental evidence ([Buchberger, 1985]) seems to indicate that this does not happen too often.

\section{Preliminary definitions}

We refer to subsets of the 2D Euclidean space as figures. Thus, the four connected black regions in Fig. 1 describe four figures. A figure can also be described by a characteristic function. The function $f\{x, y)$ is a characteristic function if $f(x, y)=1$ for points in the figure,

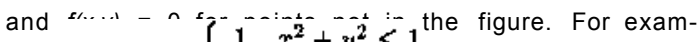
ple, $f(x, y)=\left\{\begin{array}{ll}1 & x^{2}+y^{2} \leq 1 \\ 0 & \text { otherwise }\end{array}\right.$ is the characteristic function of a circular disc of radius 1 centered at the origin.

Specifying a figure in terms of its characteristic function must be done with respect to a coordinate system. Therefore, a figure may (and usually does) change when a coordinate transformation is applied. A shape is a geometric property of a figure. Its formal definition (see, e.g., [Veblen and Whitehead, 1967]) is given in terms of properties invariant under a group of coordinate transformations.

Definition: Let $\Psi$ be a group of coordinate transformations (e.g., translations and rotations). The function $\mathrm{I}$ is invariant with respect to $\Psi$ if

$$
I(f(x, y))=I(\psi(f(x, y)))
$$

for all characteristic functions $f(x, y)$ and all transformations'0E 111.

Examples: The area of a figure is invariant under translation and rotation but not under scaling. The number of polygon edges is invariant under translation, rotation, and scaling.

Definition: A shape of a figure is a pair $\langle J, \Psi\rangle$, where $I$ is invariant under the group of coordinate transformations $\Psi$.

The two most common group transformations in the definition of shapes are translation rotation and scale (orthogonal transformations), and the general linear transformation. For example, a triangle is defined with respect to arbitrary linear transformations, but a right triangle is defined only with respect to orthogonal transformations.

\subsection{A review of moment invariants}

The classic technique for generating invariants in terms of algebraic moments was originally proposed by $\mathrm{Hu}[\mathrm{Hu}$, 1962]. The algebraic moments of the characteristic function $f(x, y)$ are defined to be:

$$
m_{p q}=\int_{x} \int_{y} x^{p} y^{q} f(x, y) d y d x
$$

These can be approximated in the discrete case by:

$$
m_{p q}=\sum_{x} \sum_{y} x^{p} y^{q} f(x, y)
$$

A figure is uniquely determined by its algebraic moments [Hu, 1962]. Therefore, instead of looking for invariants of characteristic functions one can look for invariants of moments. In practical applications only invariants of low order moments are used. (The order of the moment $m_{v q}$ is defined to be $p+q$.) Moment invariants are usually specified in terms of centralized moments, i.e., the moments measured with respect to the "center of mass":

$$
\begin{gathered}
\bar{x}=\frac{m_{10}}{m_{00}}, \quad \bar{y}=\frac{m_{01}}{m_{00}}, \\
\mu_{p q}=\int_{x} \int_{y}(x-\bar{x})^{p}(y-\bar{y})^{q} f(x, y) d y d x .
\end{gathered}
$$

The above equations can be expanded to an explicit expression giving the centralized moments in terms of the ordinary moments:

$$
\mu_{p q}=\sum_{\substack{0 \leq s \leq p \\
0 \leq t \leq q}}(-1)^{p+q-s-t}\left(\begin{array}{l}
p \\
s
\end{array}\right)\left(\begin{array}{l}
q \\
t
\end{array}\right) \frac{m_{10}^{p-s} m_{01}^{q-t}}{m_{00}^{p+q-s-t} m_{n t}}
$$

The specific formulae for the moments up to second order are:

$$
\begin{aligned}
& \mu_{00}=m_{00} \\
& \mu_{01}=\mu_{10}=0 \\
& \mu_{20}=m_{20}-m_{10}^{2} / m_{00} \\
& \mu_{11}=m_{11}-m_{10} m_{01} / m_{00} \\
& \mu_{02}=m_{02}-m_{01}^{2} / m_{00}
\end{aligned}
$$

Hu derived two second order moments invariants for translation and rotation:

$$
\begin{aligned}
& I_{1}=\mu_{20}+\mu_{02} \\
& I_{2}=\left(\mu_{20}-\mu_{02}\right)^{2}+4 \mu_{11}^{2}
\end{aligned}
$$

The centralized moments in Equation (3) can be replaced by normalized moments, $n_{p q}$, to produce invariants for translation, rotation, and scale. The normalized moments are computed from the centralized moments as follows:

$$
\eta_{p q}=\frac{\mu_{p q}}{\mu_{00}^{\gamma}}, \quad \text { with } \gamma=\frac{p+q}{2}+1
$$

The only moment invariant under general linear transformations that can be described in terms of second order moments is [Hu, 1962; Reiss, 199I]:

$$
\frac{\mu_{20} \mu_{02}-\mu_{11}^{2}}{\mu_{00}^{4}}
$$

In summary, the classic method of moment invariants can be applied to geometric shapes defined in such a way that all their instances can be generated by geometric transformations (e.g., translations, rotations, and rescaling) of a single instance. In such a case, moment invariants are computed by formulae such as those given in Equations (3) and (4), applied to the characteristic function of a single figure with the desired shape. 


\section{Invariants of shapes described in propositional logic}

It is often necessary to deal with shapes that are more complex than the simple shapes of Section 2. Suppose we are given the following shape description:

a rectangle OR (a triangle AND a polygon)

Since triangles are polygons this can be simplified to:

a rectangle OR a triangle

This simplification cannot be obtained from purely geometric knowledge because of the logic connectives. However, it cannot be obtained directly from logic since the fact that triangles are polygons requires geometric knowledge. We also observe that in this case it is impossible to calculate moment invariants from a single example shape since the moment invariants of a rectangle clearly differ from those of a triangle. In this section we show how to calculate moment invariants of shapes described in propositional logic.

\subsection{Moment invariants as a system of polynomial identities}

The central idea that enables the generalization of moment invariants to complex shapes is that moment invariants can also be viewed as a system of polynomial identities among the moments. Thus, for example, the moment invariants in (3) can also be written as:

$$
\mu_{20}+\mu_{02}-I_{1}=0, \quad\left(\mu_{20}-\mu_{02}\right)^{2}+4 \mu_{11}^{2}-I_{2}=0 .
$$

The above observation, the results of $\mathrm{Hu}[\mathrm{Hu}, 1962]$ about the relation between moment invariants and algebraic invariants, and Hilbert Basis Theorem imply the following:

Theorem: Let / be a figure. There exists a finite set of polynomial identities (among moments) that hold only for figures obtained from / by: (a) translation and rotation, (b) translation rotation and scale, (c) arbitrary linear transformations.

This suggests the following generalization of moment invariants:

Definition: Let $P=\left\{\mathrm{p} 1, \ldots, \mathrm{p}_{\mathrm{m}}\right\}$ be a set of polynomial identities among moments. We say that $P$ characterizes the shape $S$ if only the figures with the shape $S$ satisfy all the identities in $P$.

\subsection{Shapes as formulae with logic connectives}

Using logic connectives, shapes can be defined in an analogous way to formulae in propositional logic:

(i) If $S_{1}$ and $S_{2}$ are shapes then a figure / has the shape (i) $V\left(S_{2}\right)$ if it has the shape $S_{1}$ or the shape $S_{2}$.

(ii) If $S_{1}$ and $S_{2}$ are shapes then a figure / has the shape $\left(S_{1}\right)$ A $\left(S_{2}\right)$ if it has the shape $S_{1}$ and the shape $S_{2}$. (iii) If $S$ is a shape then a figure / has the shape $->(S)$ if it does not have the shape 5 .

Formulae in propositional logic created without rule (iii) are called monotone formulae. Many interesting concepts can be described by short monotone formulae [Valiant, 1984], but it is known that some short formulae may become exponentially long when described as monotone formulae [Valiant, 1983].

The moment invariants (as a set of polynomial identities) of shapes described by monotone formulae can be computed as follows. Let $P=\left\{p_{1} \ldots, p_{m}\right\}$ be the moment invariants of $S_{1}$ and let $Q=\left\{q_{1}, \ldots, q_{n}\right\}$ be the moment invariants of $S_{2}$ then:

(i) the moment invariants of $\left(S_{1}\right) \vee\left(S_{2}\right)$ are $\left\{p_{i} q_{j}: i=\right.$ $\mathrm{I}, \ldots, \mathrm{m}, j=\mathrm{I}, \ldots, \mathrm{n}\}$

(ii) the moment invariants of $\left(S_{1}\right) \wedge\left(S_{2}\right)$ are $P \cup Q$.

This defines recursively shapes described by arbitrary monotone formulae.

\section{The Grobner Basis of shape invariants}

If the system of polynomial identities describing the shape $S_{1}$ contains $m$ identities and the system of polynomial identities describing the shape $S_{2}$ contains $n$ identities, the technique of Section 3.2 produces only $m,+n$ polynomial identities for $\left(S_{1}\right)$ A $\left(S_{2}\right)$, but there are $m n$ polynomial identities for $\left(S_{1}\right) \vee\left(S_{2}\right)$. Therefore, the number of polynomial identities may grow exponentially in the size of the formula. Many of these invariants may be redundant; the redundancy can often be eliminated by representing the set of polynomial identities as a reduced Grobner base [Buchberger, 1985]. Although it is known that there are cases where the number of polynomials in the reduced Grobner base is super-exponential in the number of the original polynomials [Huynh, 1986], this does not happen too often in practice. Specifically, the Grobner basis technique was applied successfully in work on automatic theorem proving [Kapur, 1989] and robot motion planning [Cox et al., 1992]. Both applications involve similar expressions to the ones described here.

\subsection{The Grobner basis algorithm}

The Grobner basis algorithm is based on associating a fixed ordering on the moments (the variables of the polynomial identities) and then applying reductions between polynomials. The most common orderings are lexicographic and total degree. Buchberger's algorithm for the computation of a Grobner basis is based on two principal operations:

Reduction: the polynomial / can be reduced to $h$ with respect to $g$ if there is a constant $c$ such that:

$$
h=f-c \cdot g,
$$

where $c$ is specified as follows: Let $a_{g}$ be the leading monomial (with respect to the fixed ordering of variables) of $g$ then there is a monomial aj of / such that $a_{f}=c \cdot a_{g}$. 
S.Polynomial: The S.Polynomial of $f_{1} f_{2}$ is defined to be:

$$
S\left(f_{1}, f_{2}\right)=u_{1} f_{1}-\frac{c_{1}}{c_{2}} u_{2} f_{2}
$$

where $u_{1}, u_{2}, c_{1}, c_{2}$ are determined from $f_{1}, f_{2}$ as follows: Let $a_{1}, a_{2}$ be the leading monomials of $f_{1}, f_{2}$ respectively, then $c_{1}, c_{2}$ are the coefficients of $a_{1}, a_{2}$. Let $a$ be the least common multiplier of $a_{1}$ and $a_{2}$. The monomiais $u_{1}, u_{2}$ are given by: $u_{1}=a / a_{1}, u_{2}=a / a_{2}$.

Let $P$ be a set of polynomial identities. (In our case these identities define a shape.) The following algorithm calculates $G^{P}$, the Gröbner basis of $P$.

- Start with $G^{0}=P$

- Repeat while $G^{i} \neq G^{i+1}$

Take $G^{i+1}$ to be the set containing the polynomials in $G^{i}$ and the S.Polynomials of all pairs of polynomials in $G^{1}$.

$$
\text { - } G^{p}=G^{i}
$$

For complete details of the algorithm and terminution/correctness proofs see [Becker and Weispenning, I993; Buchberger, 1985]. The efficiency of this algorithm can be greatly improved; it can also be modified to compute a reduced Gröbner basis . A reduced Gröbner basis is a Gröbner basis where each polynomial is reduced with respect to all other polynomials in the basis, and all leading coefficients arc 1. In (Buchberger, 1985) it is stown that the reduced Gröbster basis is unique. Efficient algorithms for computing the reduced Gröbner basis can be found in [Becker and Weispfenning, 1993; Buchberger, 1985; Mishra and Yap, 1989].

It is known that the Grobner basis algorithm always terminates after a finite number of stepss. Unfortunately, it has also been shown that the number of resulting eleInents (and therefore, the worst case couplexity) can be super-exponential in the size of $P$ [Huynh, 1986]. Efficient implementations of the Gröbner basis algorithm ate available with many computer algebra software packages such as MATHEMATICA, REDUCE, and MAPLE.

\subsection{Gröbner basis representation and reasoning about shapes}

Invariants can be used to determine if a figure has a particular shape. This section shows how to use the Gröbner basis representation of shapes to solve in a finite numher of steps many higher level decision problems related to shapes. The ideas presented bere are closely related to work on automatic theorem proving [Chou, 1988; Kapur, 1989].

Let $S$ and $T$ be two shapes, and let $g$ be a geometric property. Consider the following questions:

a. Do $S$ and $T$ describe the exact same shape?

b. Is the shape $S$ more general than the shape $T$ ? (Does the knowledge that a figure is of shape $T$ imply that it is of shape $S$ ?)

c. Are there any figures with the shape $S$ ? d. Is a geometric property $g$ implied by $S$ ? (Does the knowledge that a figure is of shape $S$ imply that it has the geometric property $g$ ?)

e. Are there any figures with both shapes $S$ and $T$ ?

When the shapes are described in terms of polynomial identities these decision problems (and many others) can be directly translated to computational techniques utilizing the Gröbner basis representation. See (Becker and Weisptenning, 1993; Buchberger, 1985; Mishra and Yap, 1989]. We sketch the solution for the first four problems. Let $G^{S}, G^{\gamma}, G^{(s \cup T)}$, and $G^{\left(S \cup_{B}\right)}$ be the reduced Gröbner bases of the polynomial identities describing $S, T, S \cup T$, and $S \cup\{g\}$ respectively.

a. $S$ and $T$ describe the exact same shape if and only if $G^{s}=G^{T}$.

b. $S$ is more general than $T$ if and only if $G^{S}=G^{(S \cup T)}$.

c. There are no figures with the shape $S$ if and only if $l \in G^{s}$.

d. Being of shape $S$ implies the geometric property $g$ if and only if $G^{S}=G^{\left(5 \cup_{g}\right)}$.

\section{The vocabulary of low order moment invariants}

In practical applications few invariants of low order are used (see, e.g., $[\mathrm{Hu}, 1962 ; \mathrm{Li}, 1992$; Wong and Hall, 1978]). In this section we characterize the representation power of low order moment invariants and compute second order moment identities for several shapes and geometric properties.

\subsection{Representation power of low order moment invariants}

Let $\Psi$ be a group of transformations. Let $I^{k}$ be the set of moment invariants (w.r.t. $\Psi$ ) that can be computed from moments of order bounded by $k$. From the remarks in Section 3.1 it follows that $I^{\infty}$, the entire set of moment invariants, completely characterizes a shape with respect to translation and rotation. Specifically, for any two figures $A, B$ there exists a transionation involving translation and rotation that transforms $A$ to $B$ if and only if $i(A)=i(B)$ for all moment invariants $i \in I^{\infty}$.

Even though $I^{\infty}$ is finite (as implied by the Hilbert $\mathrm{B}$ sis Theorem), invariants involving high order moments may be very complex and unstable. Most practical applications use $I^{2}$ or $I^{3}$ to approximate shapes. Clearly, for a small constant $k$ the invariants in $I^{k}$ cannot cornpletely characterize shapes. Our goal is to show that they completely characterize well defined least squares approximations of shapes.

Let $A$ be a figure and let $f_{A}(x, y)$ be its characteristic function. Let $p_{A}^{k}(x, y)$ be a polynomial (in $x, y$ ) of total degree $k$ that approximates $f_{A}$ by minimizing the least squares error:

$$
E=\sum_{x, y}\left(f_{A}(x, y)-p_{A}^{k}(x, y)\right)^{2}
$$


The polynomial $p_{A}^{k}$ is known to be uniquely determined from $k$ and $f_{A}$.

Theorem: Let $A, B$ be two figures. Let $I^{k}$ be the set of moment invariants with respect to a group of transformations $\Psi$ that can be computed from moments of order bounded by $k$. Let $p_{A}^{k}, p_{B}^{k}$ be the least squares polynomial approximations to $A, B$ respectively. Then:

(i) If there is a transformation $\psi \in \Psi$ such that $\psi\left(p_{A}^{k}\right)=$ $p_{B}^{k}$ then $i(A)=i(B)$ for all $i \in I^{k}$.

(ii) If $i(A) \pm i(B)$ for all $i \in I^{k}$, and $\Psi$ is the group of translations and rotations, or the group of translations rotations and scale, then there exists a transformation $\psi \in \Psi$ such that $\psi\left(p_{A}^{k}\right)=p_{B}^{k}$.

\section{Proof:}

To keep notation tidy we write $M^{k}(g)$ for the moments of degree bounded by $k$ of a function $g$, and $I^{k}(g)$ for the moment invariants of $g$ that can be expressed in terms of $M^{k}(g)$. We need the following known facts from the theory of least squares approximations:

Fact 1: If $p$ is a least squares polynomial approximating a function $f$ and $a_{i j} x^{2} y^{j}$ is a monomial of $p$, then the $m_{i j}$ moments of $p$ and $f$ are identical. (Proof: since the derivative of the error $E$ in Equation (5) with respect to $a_{i j}$ vanishes, we have $\sum_{x, y}(f-p) x^{i} y^{j}=0$, so that $\sum_{x, y} f x^{i} y^{j}=\sum_{x, y} p x^{i} y^{j}$.)

Fact 2: Let $p_{+} q$ be two polynomials of total degree $k$.

$$
p=q \Longleftrightarrow M^{k}(p)=M^{k}(q)
$$

(Proof sketch: the polynomials of total degree $k$ form a vector space, and the moments of order bounded by $k$ are known to be a basis.)

Lemma: If $\psi$ is a linear transformation then for any figure $A$,

$$
p_{\psi(A)}^{k}=\psi\left(p_{A}^{k}\right)
$$

Proof:

$$
\begin{array}{rlr}
M^{k}\left(p_{W(A)}^{k}\right) & =M^{k}(\psi(A)) & \text { (Fact 1) } \\
& =\psi\left(M^{k}(A)\right) & \text { (linearity of } \psi) \\
& =\psi\left(M^{k}\left(p_{A}^{k}\right)\right) & \text { (Fact 1) } \\
& =M^{k}\left(\psi\left(p_{A}^{k}\right)\right) & \text { (linearity of } \psi)
\end{array}
$$

Since $\psi$ is linear, $\psi\left(p_{A}^{k}\right)$ is a polynomial of total degree $k$ and the proof is completed by applying Fact 2 to the above equality.

Proof of Part (i): We need to show that $I^{k}(A)=$ $I^{k}(B)$. From Fact 1 we have: $M^{k}(B)=M^{k}\left(p_{B}^{k}\right)$ and since $p_{B}^{k}=\psi\left(p_{A}^{k}\right)$ we have $M^{k}(B)=M^{k}\left(\psi\left(p_{A}^{k}\right)\right)$. Therefore, $I^{k}(B)=I^{k}\left(\psi\left(p_{A}^{k}\right)\right)$. From similar arguments we have $I^{k}(A)=I^{k}\left(p_{A}^{k}\right)$ and to prove Part (i) it remains to show that $I^{k}\left(p_{A}^{k}\right)=I^{k}\left(\psi\left(p_{A}^{k}\right)\right)$. This is true because by definition the expressions in $I^{k}$ are invariant under $\psi \in \Psi$.

Proof of Part (ii): Hu [Hu, 1962] shows how to "normalize" a figure with respect to translation and rotation, or translations rotations and scale. The normalization of a figure $A$ is achieved by translating and rotating $A$ to obtain $\bar{A}$ such that

$$
m_{10}(\bar{A})=m_{01}(\bar{A})=m_{11}(\bar{A})=0 .
$$

Normalization with respect to scaie is achieved by applying scaling to $\bar{A}$ so that $m_{\mathrm{Do}}(\bar{A})=1$. Hu also shows that the moments of degree bounded by $k$ of the normalized figure $\bar{A}$ uniquely determine its moment invariants and vice versa. In our notation this fact can be expressed as:

$$
\begin{gathered}
I^{k}(\bar{A})=I^{k}(\bar{B}) \Longleftrightarrow M^{k}(\bar{A})=M^{k}(\bar{B}) \\
\text { when } \bar{A}, \bar{B} \text { are "normalized" figures }
\end{gathered}
$$

To prove Part (ii) let $\bar{A}, \vec{B}$ be the normalizations of $A, B$ with

$$
\bar{A}=\psi_{1}(A), \quad B=\psi_{2}(B), \quad \psi_{1}, \psi_{2} \in \Psi
$$

We prove that $p_{A}^{k}=\left(\psi_{1}^{-1} \psi_{2}\right) p_{B}^{k}$.

$$
\begin{array}{rrr} 
& I^{k}(A)=I^{k}(B) & \text { (assumption) } \\
\Rightarrow & I^{k}(\bar{A})=I^{k}(\bar{B}) & \text { (invariance of } \left.I^{k}\right) \\
\Rightarrow & M^{k}(\bar{A})=M^{k}(\bar{B}) & (\bar{A}, \bar{B} \text { are normalized) } \\
\Rightarrow & p_{\bar{A}}^{k}=p_{\bar{B}}^{k} & \text { (farts } 1,2) \\
\Rightarrow & p_{\psi_{1}(A)}^{k}=p_{\psi_{3}(B)}^{k} & \\
\Rightarrow & \psi_{1}\left(p_{A}^{k}\right)=\psi_{z}\left(p_{B}^{k}\right) & \text { (lemma) } \\
\Rightarrow & p_{A}^{k}=\left(\psi_{1}^{-1} \psi_{2}\right) p_{B}^{k} & \text { (since } \Psi \text { is a group) }
\end{array}
$$

\subsection{What can be characterized by second order moment invariants}

The theorem in the previous section describes exactly how different shapes need to be so that they would have different finite order moment invariants. In this section we consider shapes that can be classified using invariants from $I^{2}$

Once a particular figure is given, we can compute its second order moments by using Equation (1), compute the centralized moments from Equation (2), and compute the following four numbers:

$$
\begin{aligned}
& I_{0}=\mu_{00} \\
& I_{1}=\mu_{20}+\mu_{02} \\
& I_{2}=\left(\mu_{20}-\mu_{02}\right)^{2}+4 \mu_{11}^{2} \\
& I_{3}=\mu_{20} \mu_{02}-\mu_{11}^{2}
\end{aligned}
$$

From the work of $\mathrm{Hu}[\mathrm{Hu}, 1962]$ (see also [Reiss, 1991]) it follows that the following is a complete set of algebraically independent invariant polynomials under translation and rotation for moments up to the second order:

$$
\begin{aligned}
& \mu_{00}-I_{0}=0 \\
& \mu_{20}+\mu_{02}-I_{1}=0 \\
& \left(\mu_{20}-\mu_{02}\right)^{2}+4 \mu_{11}^{2}-I_{2}=0
\end{aligned}
$$

By normalizing (6) with respect to size (se Section 2.1), the following forms a complete set of second order moment invariants under translation, rotation, and scale [Hu, 1962; Reiss, 1991]:

$$
\begin{aligned}
& \mu_{20}+\mu_{02}-I_{1} \mu_{00}^{2}=0 \\
& \left(\mu_{20}-\mu_{02}\right)^{2}+4 \mu_{11}^{2}-I_{2} \mu_{00}^{4}=0
\end{aligned}
$$


Under a general linear transformation, the following invariant is the complete set of polynomial identities (up to second order) [Hu, 1962; Reiss, 1991 :

$$
\mu_{20} \mu_{02}-\mu_{11}^{2}-I_{3} \mu_{00}^{4}=0
$$

A figure has $n$-fold rotational symmetry if rotating it by $2 \pi / \pi$ radians around its center leaves it unchanged. The property of 4-fold rotational symmetry can be characterized by second order moment invariants as follows:

$$
I_{2}(A)=0 \Longleftrightarrow A \text { has } 4 \text {-fold rotational symmetry. }
$$

Equation (9) follows from the fact that normalized figures with 4-fold rotational symmetry must have $\mathrm{m}_{20}=$ $\mathrm{m}_{02^{-}}$Since they also have $\mathrm{m}_{11}=0$ it follows from Equation (6) that $I_{2}=0$.

\subsection{Moment Identities of specific shapes and properties}

In this section moment identities are computed for several shapes. They are based on moment values of the specific black figures shown on the left, which were computed via Equation (1) (using definite integration).

A triangle is invariant under general linear transformations. Therefore, it can be characterized in terms of the polynomial in (8). Computing the value of $I_{3}$ for the triangle figure on the left gives $I_{3}=1 / 108$. Thus, The least squares approximation of a triangle by a polynomial of total degree two is characterized by the following moment identity:

$$
\mu_{20} \mu_{02}-\mu_{11}^{2}-\frac{1}{108} \mu_{00}^{4}=0
$$

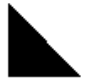

Since the shape of a right triangle is invariant under translation, rotation, and scale, it can be characterized in terms of the polynomial identities in (7). Computing the value of $I_{1}, I_{2}$ for the right triangle on the left gives $I_{1}=\frac{2}{9}, I_{2}=\frac{1}{81}$. Thus, the least squares approximation of a right triangle by a polynomial of total degree two is characterized by the following moment identities:

$$
\begin{aligned}
& \mu_{20}+\mu_{02}-\frac{2}{9} \mu_{00}^{2}=0 \\
& \left(\mu_{20}-\mu_{02}\right)^{2}+4 \mu_{11}^{2}-\frac{1}{81} \mu_{00}^{4}=0
\end{aligned}
$$

A rectangle (actually a quadrilateral) is invariant under general linear transformations. It can be characterized in terms of the polynomial in (8). Computing the value of $I_{3}$ for the rectangle on the left gives $I_{\mathbf{3}}=\frac{1}{144}$. Hence, a least squares approximation of a rectangle (quadrilateral) by a polynomial of total degree two is given by the following moment identity:

$$
\mu_{20} \mu_{02}-\mu_{11}^{2}-\frac{1}{144} \mu_{00}^{4}=0
$$

A square is invariant under translation rotation and scale. Therefore, it can be characterized in terms of the polynomials in (7). Computing the value $I_{1} I_{2}$ for the square on the left gives $I_{1}=\frac{1}{a}, I_{2}=0$. Thus, the least squares approximation of a square by a polynomial of total degree two is given by the following moment identities:

$$
\begin{aligned}
& \mu_{20}+\mu_{02}-\frac{1}{6} \mu_{00}^{2}=0 \\
& \left(\mu_{20}-\mu_{02}\right)^{2}+4 \mu_{11}^{2}=0
\end{aligned}
$$

OR

This shape is described as being a rectangle OR a square, and can be characterized by using Equation (12) for a rectangle, Equations (13) for a square, and applying the rules of section 3.2. This gives the following set of moment identities:

$$
\begin{aligned}
& \left(\mu_{20} \mu_{02}-\mu_{11}^{2}-\frac{1}{144} \mu_{00}^{4}\right)\left(\mu_{20}+\mu_{02}-\frac{1}{6} \mu_{00}^{2}\right)=0 \\
& \left(\mu_{20} \mu_{02}-\mu_{11}^{2}-\frac{1}{144} \mu_{00}^{4}\right)\left(\left(\mu_{20}-\mu_{02}\right)^{2}+4 \mu_{11}^{2}\right)=0
\end{aligned}
$$

We show in the next section that these identities can be reduced to the identity of a rectangle by using the Grobner basis technique.

AREA. The area of a shape is invariant under translation and rotation, and is characterized by $I_{0}$. For example, a shape having an area of 10 is characterized by the following moment identity:

$$
\mu_{00}-10=0
$$

ROTATIONAL SYMMETRY. The characterization of 4 -fold rotational symmetry by Equation 9 gives the following identity:

$$
\left(\mu_{20}-\mu_{02}\right)^{2}+4 \mu_{11}^{2}=0
$$

\section{Examples utilizing the Grobner basis representation}

The identities from the previous section are now used to describe examples of the Grobner basis algorithmic approach to reasoning about shapes, as described in Section 4.2. Unless otherwise stated, a reference to a shape in this section is to be understood as a reference to a least squares approximation of the shape with a polynomial of total degree 2. The examples here correspond to the problems outlined in Section 4.2.

To show that a rectangle with 4-fold rotational symmetry is a square we verify that the reduced Grobner basis of identities (14),(12) is the same as the reduced Grobner basis of (13).

\begin{tabular}{|c|c|}
\hline Moment Identities & Reduced bayis \\
\hline$\mu_{20} \mu_{02}-\mu_{11}^{2}-\frac{1}{144} \mu_{00}^{4}$ & $\mu_{20}+\mu_{02}-\frac{1}{6} \mu_{00}^{2}$ \\
$\left(\mu_{20}-\mu_{02}\right)^{2}+4 \mu_{12}^{2}$ & $\left(\mu_{20}-\mu_{02}\right)^{2}+4 \mu_{12}^{2}$ \\
\hline
\end{tabular}

To show that a rectangle is more general than a square we show that the reduced Grobner basis of (12) is the same as the reduced Grobner basis created from the identities (12),(13).

\begin{tabular}{|l|c|}
\hline \multicolumn{1}{|c|}{ Moment Identities } & Reduced basis \\
\hline$\mu_{20} \mu_{02}-\mu_{11}^{2}-\frac{1}{144} \mu_{00}^{4}$ & \\
$\mu_{20}+\mu_{02}-\frac{1}{6} \mu_{00}^{2}$ & $\mu_{20} \mu_{02}-\mu_{11}^{2}-\frac{1}{144} \mu_{00}^{4}$ \\
$\left(\mu_{20}-\mu_{02}\right)^{2}+4 \mu_{14}^{2}$ & \\
\hline
\end{tabular}


To show that there exists a rectangle of size 30 with 4fold rotational symmetry we compute the Gröbner basis of the identities in equations (14),(12) with the additional equation $\mu_{00}=30$. Since the Gröbner basis does not contain l, such a shape exists.

\begin{tabular}{|l|l|}
\hline \multicolumn{1}{|c|}{ Moment Identities } & \multicolumn{1}{c|}{ Reduced basis } \\
\hline$\mu_{20} \mu_{02}-\mu_{11}^{2}-\frac{1}{144} \mu_{00}^{4}$ & $\mu_{00}-30$ \\
$\mu_{00}-30$ & $\mu_{20}+\mu_{02}-150$ \\
$\left(\mu_{20}-\mu_{02}\right)^{2}+4 \mu_{11}^{2}$ & $\left(\mu_{20}-\mu_{02}\right)^{2}+4 \mu_{11}^{2}$ \\
\hline
\end{tabular}

To show that a square has 4-fold rotational we need to show that the reduced Grobner basis of the identities in (14),(13) is the same as the Gröbner basis of the identities in (13).

\begin{tabular}{|c|c|}
\hline Moment Identities & Reduced basis \\
\hline$\mu_{20}+\mu_{02}-\frac{1}{6} \mu_{00}^{2}$ & $\mu_{20}^{2}+\mu_{02}-\frac{1}{6} \mu_{00}^{2}$ \\
$\left(\mu_{20}-\mu_{02}\right)^{2}+4 \mu_{11}^{2}$ & $\left(\mu_{20}-\mu_{02}\right)^{2}+4 \mu_{11}^{2}$ \\
$\left(\mu_{20}-\mu_{02}\right)^{2}+4 \mu_{11}^{2}$ & \\
\hline
\end{tabular}

To show that there are no right triangles of size 10 with 4-fold rotational symmetry we show the Gröbner basis of the identities in equations (14),(10), with the additional equation $\mu_{00}=10$ reduces to 1 .

\begin{tabular}{|c|c|}
\hline Moment Identities & Reduced basis \\
\hline$\mu_{20}+\mu_{02}-\frac{2}{8} \mu_{00}^{2}$ & \\
$\left(\mu_{20}-\mu_{02}\right)^{2}+4 \mu_{11}^{4}-\frac{1}{81} \mu_{00}^{4}$ & 1 \\
$\left(\mu_{20}-\mu_{02}\right)^{2}+4 \mu_{11}^{2}$ & \\
$\mu_{00}-10$ & \\
\hline
\end{tabular}

To show that the shape described as:

\section{(rectangle AND 4-fold rotational symmetry) OR square}

reduces to a square, we build the moment identities following the procedure of Section 3.2 and show the reduced Gröbner basis to be the identities of a square.

\begin{tabular}{|c|}
\hline Moment Identities \\
\hline$\left(\mu_{20} \mu_{02}-\mu_{11}^{2}-\frac{1}{144} \mu_{00}^{4}\right)\left(\mu_{20}+\mu_{02}-\frac{1}{6} \mu_{00}^{2}\right)$ \\
$\left(\mu_{20} \mu_{02}-\mu_{11}^{2}-\frac{1}{144} \mu_{00}^{4}\right)\left(\left(\mu_{20}-\mu_{02}\right)^{2}+4 \mu_{11}^{2}\right)$ \\
$\left(\left(\mu_{20}-\mu_{02}\right)^{2}+4 \mu_{11}^{2}\right)\left(\mu_{20}+\mu_{02}-\frac{1}{6} \mu_{00}^{2}\right)$ \\
$\left(\left(\mu_{20}-\mu_{02}\right)^{2}+4 \mu_{11}^{2}\right)\left(\left(\mu_{20}-\mu_{02}\right)^{2}+4 \mu_{11}^{2}\right)$ \\
\hline
\end{tabular}

\begin{tabular}{|c|}
\hline Reduced basis \\
\hline$\mu_{20}+\mu_{02}-\frac{1}{6} \mu_{00}^{2}$ \\
$\left(\mu_{20}-\mu_{02}\right)^{2}+4 \mu_{12}^{2}$ \\
\hline
\end{tabular}

\section{Concluding remarks}

A simple generalization of the idea of moment invariants enables computing moment invariants of shapes described by formulace in propositional logic. Our technique is limited to monotone formulae. The representation power of low order moment invariants was shown to be directly related to least squares approximations of figures by polynomials of low degree.

The representation of invariants in a Gröbner basis enables reasoning about shapes in a way similar to geometric theorem proving techniques. The advantage of our technique over geometric theorem proving (e.g., [Chou, 1988; Kapur, 1989]) is that in our technique there is no need to explicitly describe the basic shapes. Instead, their description is extracted automatically in terms of moments. For example, a theorem prover can easily deduce that a square is a special case of a rectangle if it is given the definition of a square as a rectangle with even sides. Our technique does not require this information.

The fact that our technique uses finite measurements (moments) means that positive conclusions (e.g., the given figure is a rectangle) can only be verified for least squares approximations of the figure. On the other hand, negative conclusions (e.g., the given figure is not a rectangle) is verified with certainty.

\section{References}

[Becker and Weispfenning, 1993] T. Becker and V. Weispfenning. Grobner Bases: A Computational Approach to Commutative Algebra. Springer-Verlag, 1993.

[Buchberger, 1985] B. Buchberger. An algorithmic method in polynomial ideal theory. In N. K. Bose, editor, Multidimensional Systems Theory, pages 184-232. Reidel, 1985.

[Chou, 1988] S. C. Chou. Mechanical Geometry Theorem Proving. D. Ridel Publishing Company, 1988.

[Cox et al, 1992] D. Cox, J. Little, and D. O'Shera. Ideals, Varieties and Algorithms: An Introduction of Computational Algebraic Geometry and Commutative Algebra. Springer-Verlag, New York, 1992.

[Hu, 1962] M. Hu. Visual pattern recognition by moment invariants. IRE Trans. Inform. Theory, 8:179-187, 1962.

[Huynh, 1986] D. T. Huynh. A supercxponential lower bound for Grobner bases and the Church-Rosser commutative Thue systems. Information and Control, 68(1-3):196206, February/March 1986.

[Kapur, 1989] D. Kapur. A refutational approach to geometry theorem proving. In Deepak Kapur and Joseph L. Mundy, editors, Geometric Reasoning, pages 61-93. The MIT Press, 1989.

[Li, 1992] Yajun Li. Reforming the theory of invariant moments for pattern recognition. Pattern Recognition, 25(7):723-730, 1992.

[Mishra and Yap, 1989] B. Mishra and Chee Yap. Notes on Grobner bases. Information Sciences, 48:219-252, 1989.

[Reiss, 1991] T. H. Reiss. The revised fundamental theorem of moment invariants. IEEE Transactions on Pattern Analysis and Machine Intelligence, 13(8):830-834, August 1991.

[Valiant, 1983] L. G. Valiant. Exponential lower bounds for restricted monotone circuits. In Proceedings of the annual ACM symposium on theory of computing (STOC'83), pages 110-117, Boston, 1983.

[Valiant, 1984] L. G. Valiant. Short monotone formulae for the majority function. Journal of Algorithms, 5:363-366, 1984.

[Veblen and Whitehead, 1967] O. Veblen and J. Whitehead. The Foundations of Differential Geometry. Cambridge University Press, 1967.

[Wong and Hall, 1978] R. Y. Wong and E. L. Hall. Scene matching with invariant moments. Computer Graphics and Image Processing, 8:16-24, September 1978. 